\title{
CONTROLE DE MOVIMENTOS VOLUNTÁRIOS NO MEMBRO SUPERIOR NÃO PLÉGICO DE PORTADORES DE PARALISIA CEREBRAL HEMIPLÉGICA ESPÁSTICA
}

\author{
Barela AMF ${ }^{1}$ e Almeida GL ${ }^{2}$ \\ ${ }^{1}$ Laboratório para Estudos do Movimento, Instituto de Biociências, Universidade Estadual Paulista, Rio Claro, SP - \\ Brasil \\ ${ }^{2}$ Curso de Fisioterapia, Universidade de Ribeirão Preto, Ribeirão Preto, SP - Brasil \\ Correspondência para: Ana Maria Forti Barela, Laboratório para Estudos do Movimento, Depto de Educação Física, \\ Instituto de Biociências, UNESP, Av. 24-A,1515, Bela Vista, CEP 13506-900, Rio Claro, SP - Brasil, \\ e-mail: ambarela@gmail.com
}

Recebido: 05/12/2005 - Aceito: 22/05/2006

\begin{abstract}
RESUMO
Objetivo: Analisar os movimentos de flexão do ombro e do cotovelo do hemicorpo não plégico de portadores de paralisia cerebral (PC) hemiplégica espástica e do hemicorpo mais utilizado de indivíduos sem comprometimento motor e/ou neurológico (normais). Métodos: Seis portadores de PC (18,2 \pm 3,7 anos) e seis indivíduos normais (18,5 \pm 3,3 anos) participaram deste estudo. Marcadores ativos foram afixados nos centros articulares do ombro, cotovelo e punho para aquisição de dados cinemáticos e eletrodos de superfície foram afixados nos ventres musculares do deltóide anterior, deltóide posterior, bíceps braquial e tríceps braquial para aquisição da atividade eletromiográfica (EMG) muscular. Os participantes tiveram que flexionar o ombro na tarefa de flexão do ombro e o cotovelo na tarefa de flexão do cotovelo o mais rápido possível em direção a um alvo que estava posicionado em três distâncias angulares diferentes $\left(25^{\circ}, 50^{\circ}\right.$ e $\left.75^{\circ}\right)$. Resultados: Todos os participantes realizaram as duas tarefas motoras e os dois grupos movimentaram predominantemente o ombro para realizar a tarefa de flexão de ombro. Porém, para realizar a tarefa de flexão de cotovelo, os portadores de PC movimentaram o cotovelo e o ombro, enquanto que os indivíduos normais movimentaram predominantemente o cotovelo. Conclusão: Diferentemente dos indivíduos normais, os portadores de PC hemiplégica espástica não controlaram os movimentos mais distais da mesma forma que os movimentos proximais. Portanto, o hemicorpo não plégico não deve ser considerado normal e, mais importante, ao se propor um programa de intervenção para os portadores de PC, os dois hemicorpos devem ser considerados.
\end{abstract}

Palavras-chave: controle motor, cinemática, cinética, eletromiografia, estratégia insensível à velocidade.

\section{ABSTRACT \\ Control of Voluntary Movements in the Non-Affected Upper Limb of Spastic Hemiplegic Cerebral Palsy Patients}

Objective: To analyze shoulder and elbow flexion movements in the non-affected side of the body in spastic hemiplegic cerebral palsy (CP) patients and in the side of the body used more often in normal individuals (without motor and/or neurological impairment). Method: Six CP patients (18.2 \pm 3.7 years old) and six normal individuals (18.5 \pm 3.3 years old) participated in this study. Active markers were affixed to the shoulder, elbow and wrist joint centers in order to acquire kinematic data, and surface electrodes were affixed to the muscle belly of the anterior deltoid, posterior deltoid, biceps brachii, and triceps brachii in order to acquire muscle electromyographic data. All participants were asked to flex their shoulder joint in a shoulder flexion task and their elbow joint in an elbow flexion task, as fast as possible, towards a target that was positioned at three different angular distances $\left(25^{\circ}, 50^{\circ}\right.$ and $\left.75^{\circ}\right)$. Results: All participants accomplished both motor tasks and both groups predominantly moved the shoulder to perform the shoulder flexion task. However, to perform the elbow flexion task, the CP patients moved their elbow and shoulder, while the normal individuals predominantly moved the elbow. Conclusion: Differing from normal individuals, spastic hemiplegic CP patients did not control distal movements in the same way as done for proximal movements. Therefore, the non-affected side of the body should not be considered normal and, most importantly, both the affected and non-affected sides of the body must be taken in account in any intervention program.

Key words: motor control, kinematics, kinetics, electromyography, speed-insensitivity strategy. 


\section{INTRODUÇÃO}

Na maioria dos estudos sobre portadores de paralisia cerebral (PC) hemiplégica, a atenção é voltada para o hemicorpo plégico. Em muitos casos, é comum a comparação entre o hemicorpo plégico e o não plégico, ao invés de se comparar os portadores de PC com indivíduos sem comprometimento neurológico e/ou motor. No entanto, pode ser observado clinicamente que portadores de PC hemiplégica apresentam déficits motores acentuados no hemicorpo não plégico ${ }^{1,2}$. Uma vez que o hemicorpo não plégico é utilizado na maioria das tarefas que envolvem apenas um lado do corpo, seria interessante examinar atentamente como portadores de PC hemiplégica controlam os movimentos desse hemicorpo.

Uma possibilidade para se investigar os mecanismos de controle motor do hemicorpo não plégico de portadores de PC hemiplégica seria por meio de movimentos uniarticulares que, apesar de simples, poderiam auxiliar no entendimento de como os movimentos são controlados pelo sistema nervoso. Os movimentos uniarticulares podem ser entendidos como movimentos em uma articulação que não são acompanhados por movimentos de amplitudes comparáveis em outras articulações ${ }^{3}$. Sendo assim, para realizar um movimento de flexão de cotovelo, por exemplo, pode ocorrer uma pequena movimentação na articulação do ombro e mesmo assim o movimento é considerado uniarticular. Os movimentos uniarticulares podem ser descritos com base em variáveis cinéticas e cinemáticas, que descrevem suas características mecânicas, e com base em variáveis eletromiográficas, que descrevem os padrões de atividade muscular, por exemplo. As características mecânicas dos movimentos uniarticulares podem ser observadas em movimentos de diferentes amplitudes e velocidades ${ }^{4,5}$, sendo que existem correlações entre as características mecânicas e os padrões de atividade muscular.

Um pressuposto teórico, denominado "hipótese da estratégia dual”, que pode ser utilizado para investigar movimentos uniarticulares, foi proposto por Gottlieb et al ${ }^{6}$. De acordo com esse pressuposto, os movimentos são controlados por duas estratégias diferentes: a primeira estratégia, "estratégia insensível à velocidade”, se refere aos movimentos que podem ser realizados o mais rápido possível ${ }^{5,6}$, e a segunda estratégia, "estratégia sensível à velocidade”, se refere aos movimentos em que a velocidade é explicitamente controlada pelo executante ${ }^{4,6}$. O pressuposto da hipótese da estratégia dual foi utilizado para verificar vários aspectos de controle motor em indivíduos sem qualquer comprometimento motor e/ou neurológico ${ }^{7}$, em portadores da Síndrome de Down ${ }^{8}$, em portadores de PC $^{9}$, entre outros, e foi escolhido para investigar movimentos uniarticulares de portadores de PC hemiplégica espástica no presente estudo.

Sendo assim, o objetivo deste estudo foi de analisar os movimentos das articulações do ombro e do cotovelo do hemicorpo não plégico de portadores de PC e do hemicorpo mais utilizado de indivíduos sem comprometimento motor e/ou neurológico durante a execução das tarefas de flexão de ombro e de flexão de cotovelo. Mais especificamente, variáveis cinemáticas, cinéticas e eletromiográficas foram analisadas com base no pressuposto da hipótese da estratégia dual ${ }^{6}$.

\section{MÉTODOS}

\section{Participantes}

Seis portadores de PC hemiplégica espástica (que desse ponto em diante serão tratados como portadores de PC), com idade entre 15 e 24 anos (18,2 \pm 3,7 anos) e seis indivíduos sem qualquer comprometimento motor e/ou neurológico (que desse ponto em diante serão tratados como indivíduos normais), com idade entre 15 e 23 anos (18,5 \pm 3,3 anos), participaram do presente estudo. Para participar, os portadores de PC tinham que ser capazes de entender as informações referentes às tarefas motoras e de realizá-las com o hemicorpo não plégico, e cada indivíduo normal tinha que apresentar a idade aproximada e o gênero correspondente a cada um dos portadores de PC. Todos os participantes (no caso dos menores de idade, seus responsáveis legais) assinaram um termo de consentimento aprovado pelo Comitê de Ética em Pesquisa da Faculdade de Ciências Médicas/ UNICAMP (parecer no 213/99).

\section{Procedimentos}

Os participantes tiveram que realizar duas tarefas motoras, uma de flexão de ombro e uma de flexão de cotovelo em direção a um alvo que estava disposto em três distâncias angulares diferentes, sendo ambas no plano sagital e enquanto permaneciam sentados confortavelmente em uma cadeira com o tronco ereto. $\mathrm{O}$ alvo foi posicionado de modo que as amplitudes de movimento de cada uma das articulações fossem $25^{\circ}, 50^{\circ}$ e $75^{\circ}$, respectivamente, a partir de uma da posição inicial, em que o braço permanecia ao longo do tronco e da lateral da cadeira. Para realização das tarefas, os participantes usaram uma tala de lona e com fechos de velcro para imobilizar o movimento do punho, uma vez que o movimento dessa articulação não foi investigado neste estudo. Uma ponteira de madeira (40 X 0,5 cm, comprimento e diâmetro, respectivamente) foi afixada nessa tala, de modo que os alvos fossem tocados por ela.

Para aquisição dos dados cinemáticos durante a execução das tarefas, marcadores ativos, que emitem sinais infravermelhos, foram afixados nos centros articulares do ombro, cotovelo e punho. Esses dados foram adquiridos por um sistema optoeletrônico (Optotrak 3020, NDI), com freqüência de amostragem de $200 \mathrm{~Hz}$. Para aquisição dos dados eletromiográficos, eletrodos de superfície foram afixados no ventre muscular do bíceps braquial, tríceps braquial, deltóide anterior e deltóide posterior, no sentido das fibras musculares. A região muscular em que cada eletrodo foi afixado foi limpa com 
algodão embebido em álcool. Esses dados foram adquiridos por um sistema de coleta e análise de dados eletromiográficos (EMGworks, DELSYS), com uma freqüência de amostragem de $1000 \mathrm{~Hz}$. Por fim, os dados cinemáticos e os eletromiográficos foram sincronizados por meio de um sistema de sincronização (Odau II, Optotrak Data Aquisition Unit).

Cada participante realizou um bloco de 11 repetições em cada distância angular e em cada uma das tarefas, o que totalizou 66 repetições. A ordem de cada bloco foi aleatória, e só teve início após algumas tentativas práticas para familiarização com a tarefa. No decorrer das aquisições, houve intervalo entre os blocos de repetições e, quando necessário, entre as repetições. Os dados de cada repetição foram adquiridos durante dois segundos e após esse tempo, os participantes retornavam à posição inicial.

Todos os participantes foram instruídos a realizar cada tarefa o mais rápido possível após um comando dado pelo experimentador, e para flexionar somente a articulação da tarefa selecionada: articulação do ombro para a tarefa de flexão do ombro, articulação do cotovelo para a tarefa de flexão do cotovelo. Os portadores de PC realizaram as tarefas com o braço não plégico e os indivíduos normais as realizaram com o braço direito, uma vez que era o braço mais utilizado por eles.

Após a aquisição de todas as repetições, uma aquisição de contração voluntária máxima isométrica (CVMI) durante três segundos foi realizada para cada músculo investigado. Esses dados foram utilizados para normalizar os dados eletromiográficos. Na seqüência, massa corporal e comprimento dos segmentos braço e antebraço do hemicorpo investigado foram verificados para cada participante.

\section{Análise dos dados}

Uma descrição mais detalhada dessa análise foi apresentada anteriormente ${ }^{10}$. Em termos gerais, a análise de todos os dados foi feita com a utilização do programa Matlab 4.2 (Mathworks Inc.). A primeira repetição de cada bloco foi eliminada e as demais foram analisadas. Com base nos dados cinemáticos, o deslocamento angular de cada articulação para cada tarefa foi calculado a partir das coordenadas obtidas dos marcadores ativos posicionados nos centros articulares. A velocidade e a aceleração angulares dessas articulações foram calculadas a partir do deslocamento angular. Ainda, a amplitude de movimento articular (ADM), que correspondeu à diferença dos ângulos articulares entre as posições final e inicial, e o valor máximo da velocidade angular foram calculados.

Os parâmetros inerciais (massa, distância proximal do centro de massa e momentos de inércia do centro de massa dos segmentos braço e antebraço) foram estimados a partir das medidas referentes à massa corporal e ao comprimento dos dois segmentos corporais dos participantes (para maiores detalhes, ver Winter ${ }^{11}$ ). O torque muscular das articulações do ombro e do cotovelo foi calculado a partir dos dados cinemáticos e dos parâmetros inerciais e, a partir desse torque, os impulsos de aceleração e desaceleração foram calculados como a área da curva do torque muscular pelo tempo durante as fases positivas e negativas, respectivamente.

Os dados eletromiográficos foram filtrados com o filtro Butterworth de $4^{a}$ ordem e com um filtro passa-banda de 20 $400 \mathrm{~Hz}$. Esses dados também foram retificados e filtrados novamente com um filtro de passa-baixa de $5 \mathrm{~Hz}$ para se obter o envoltório linear. Três pontos de referência foram identificados visualmente nesse envoltório, em que o primeiro ponto correspondeu ao início da atividade eletromiográfica (EMG) da musculatura agonista, o segundo ponto correspondeu ao final da atividade EMG da musculatura agonista e o terceiro ponto correspondeu ao início da atividade EMG da musculatura antagonista. A atividade EMG das musculaturas agonistas (bíceps braquial e deltóide anterior) foram integradas a partir do primeiro ponto de referência e durante 30 milissegundos para obtenção da intensidade de ativação da musculatura agonista $\left(\mathrm{Q}_{30}\right)$, e do primeiro ao segundo ponto de referência para obtenção da quantidade de atividade muscular agonista $\left(Q_{a g}\right)$. A diferença temporal entre o primeiro e o terceiro evento consistiu da latência antagonista $\left(\mathrm{L}_{\mathrm{ant}}\right)$. Finalmente, os dados eletromiográficos foram normalizados pela CVMI para possibilitar a comparação entre os participantes e as amplitudes de movimento das tarefas.

\section{Análise estatística}

Para comparar os fatores grupos (PC e N) e distâncias angulares $\left(25^{\circ}, 50^{\circ}\right.$ e $\left.75^{\circ}\right)$, cinco MANOVAs e três ANOVAs foram realizadas para cada tarefa (flexão do ombro e flexão do cotovelo), sendo que o fator distância foi tratado como medida repetida. As variáveis dependentes para a tarefa de flexão do ombro foram ADM do ombro e velocidade máxima do ombro para a primeira MANOVA; impulso de aceleração e impulso de desaceleração do ombro e do cotovelo para a segunda e a terceira MANOVAs, respectivamente; $\mathrm{Q}_{30}$ e $\mathrm{Q}_{\mathrm{ag}}$ dos músculos bíceps braquial e deltóide anterior para a quarta e a quinta MANOVAs, respectivamente; ADM do cotovelo para a primeira ANOVA; $\mathrm{L}_{\text {ant }}$ entre os músculos bíceps braquial e tríceps braquial e entre os músculos deltóide anterior e deltóide posterior para a segunda e terceira ANOVAs, respectivamente. As mesmas variáveis dependentes foram analisadas para a tarefa de flexão do cotovelo, exceto a velocidade máxima que foi a do cotovelo ao invés do ombro. Quando os fatores indicaram diferenças nas análises multivariadas, análises univariadas foram empregadas e indicaram diferença para distância angular e para interação entre grupo e distância angular, testes post hoc de Tukey para comparações pareadas $^{12}$ foram utilizados. Em todas as análises, o nível de significância foi mantido em 0,05 e o programa SPSS 10.0 (SPSS, Inc.) foi utilizado para a análise estatística. 


\section{RESULTADOS}

Todos os participantes foram capazes de realizar as tarefas de flexão do ombro e de flexão do cotovelo em direção ao alvo nas três distâncias angulares (25, 50 e $\left.75^{\circ}\right)$. A seguir, os resultados encontrados no presente estudo são apresentados separadamente para cada tarefa.

\section{Tarefa de flexão do ombro}

A Figura 1 ilustra os deslocamentos e as velocidades angulares, os torques musculares e os padrões de atividade EMG muscular referentes ao ombro (porção esquerda) e ao cotovelo (porção direita) durante a tarefa de flexão do ombro. Qualitativamente, e em termos gerais, as trajetórias cinemáticas do ombro (deslocamento e velocidade angulares) aumentaram conforme o aumento da distância angular dos alvos. A velocidade do ombro apresentou formato de sino, com pico único (velocidade máxima), e pareceu ser menor para os portadores de PC do que para os indivíduos normais. O padrão do torque muscular do ombro foi caracterizado por um impulso de aceleração e um de desaceleração e parece ter aumentado com o aumento da distância angular para o portador de PC e para o indivíduo normal. Com relação ao padrão EMG do músculo deltóide anterior, a intensidade de ativação foi constante e a quantidade de atividade EMG aumentou com o aumento da distância angular para os dois indivíduos. Apesar de a instrução ter sido para movimentar o ombro nessa tarefa, os indivíduos não foram capazes de manter o cotovelo imóvel, como pode ser observado na Figura 1 (porção direita).

A Tabela 1 apresenta os valores médios ( \pm desvio padrão) das variáveis analisadas. A ADM do ombro e a velocidade máxima aumentaram com o aumento da distância angular para os dois grupos, e a ADM do cotovelo não apresentou qualquer diferença entre os grupos e entre as distâncias angulares. Enquanto que o impulso de aceleração do ombro e do cotovelo aumentou com o aumento da distância angular para os dois grupos, o impulso de desaceleração do ombro não foi diferente entre as distâncias angulares e do cotovelo foi diferente somente entre as distâncias angulares de $25^{\circ}$ e $75^{\circ}$.

Em termos de atividade EMG dos músculos investigados, $\mathrm{Q}_{30}$ do deltóide anterior e do bíceps braquial foi a mesma nas três distâncias angulares, e $\mathrm{Q}_{\mathrm{ag}}$ do deltóide anterior aumentou com o aumento da distância angular, enquanto que do bíceps braquial não foi diferente entre as distâncias. Por fim, os portadores de PC apresentaram $\mathrm{L}_{\text {ant }}$ entre os músculos deltóide anterior e deltóide posterior menor que os indivíduos normais, enquanto que entre os músculos bíceps braquial e tríceps braquial não apresentaram diferença (Tabela 1).

Tabela 1. Média ( \pm desvio padrão) das variáveis amplitude de movimento do ombro e do cotovelo, velocidade máxima do ombro, impulsos de aceleração e de desaceleração do ombro e do cotovelo, intensidade de ativação e quantidade de atividade muscular do deltóide anterior e do bíceps braquial, e latência antagonista entre os músculos deltóide anterior e deltóide posterior, e bíceps braquial e tríceps braquial durante a tarefa de flexão de ombro dos portadores de PC e dos indivíduos normais nas três distâncias angulares $\left(25^{\circ}, 50^{\circ}\right.$ e $\left.75^{\circ}\right)$.

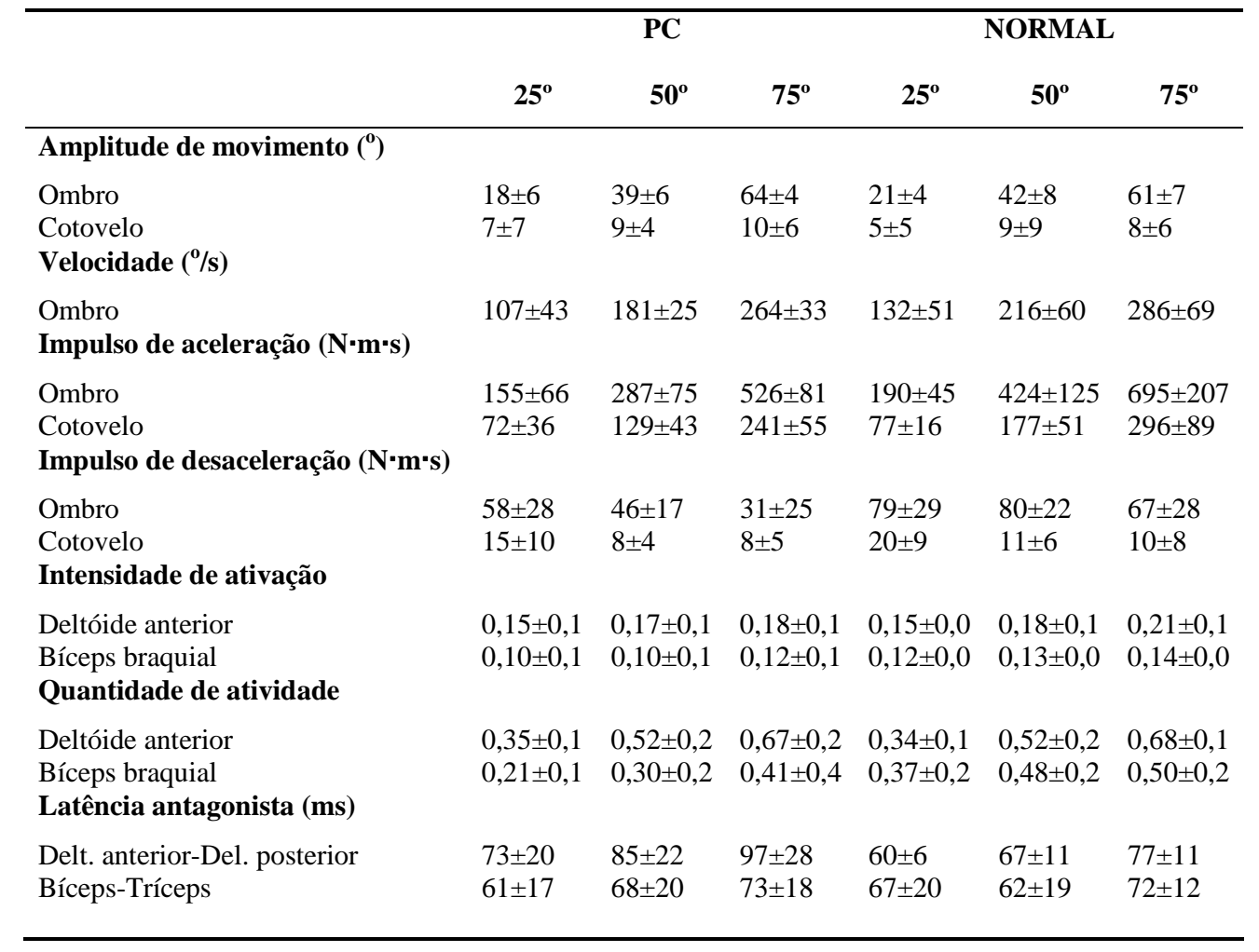


(A) Ombro
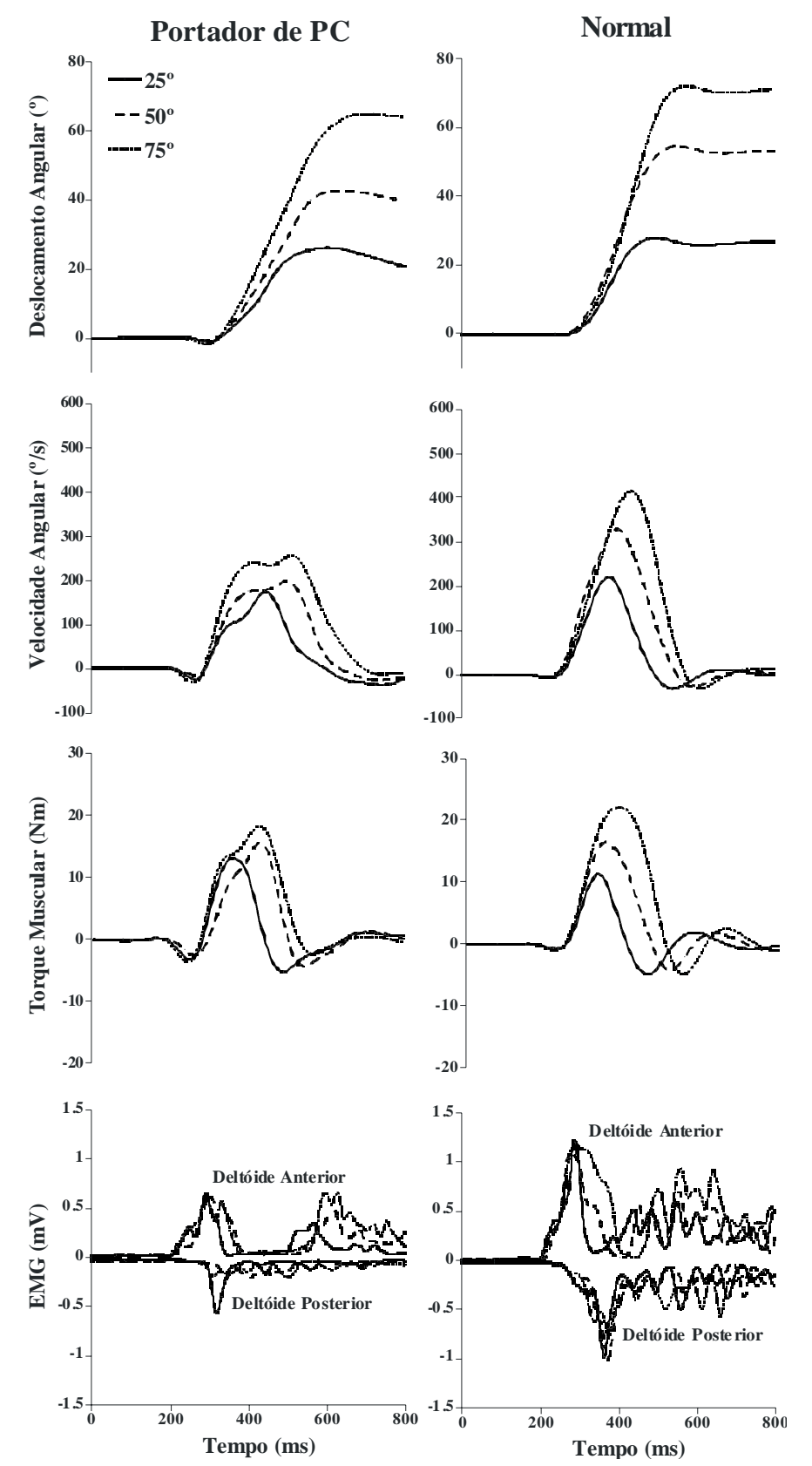

(B) Cotovelo
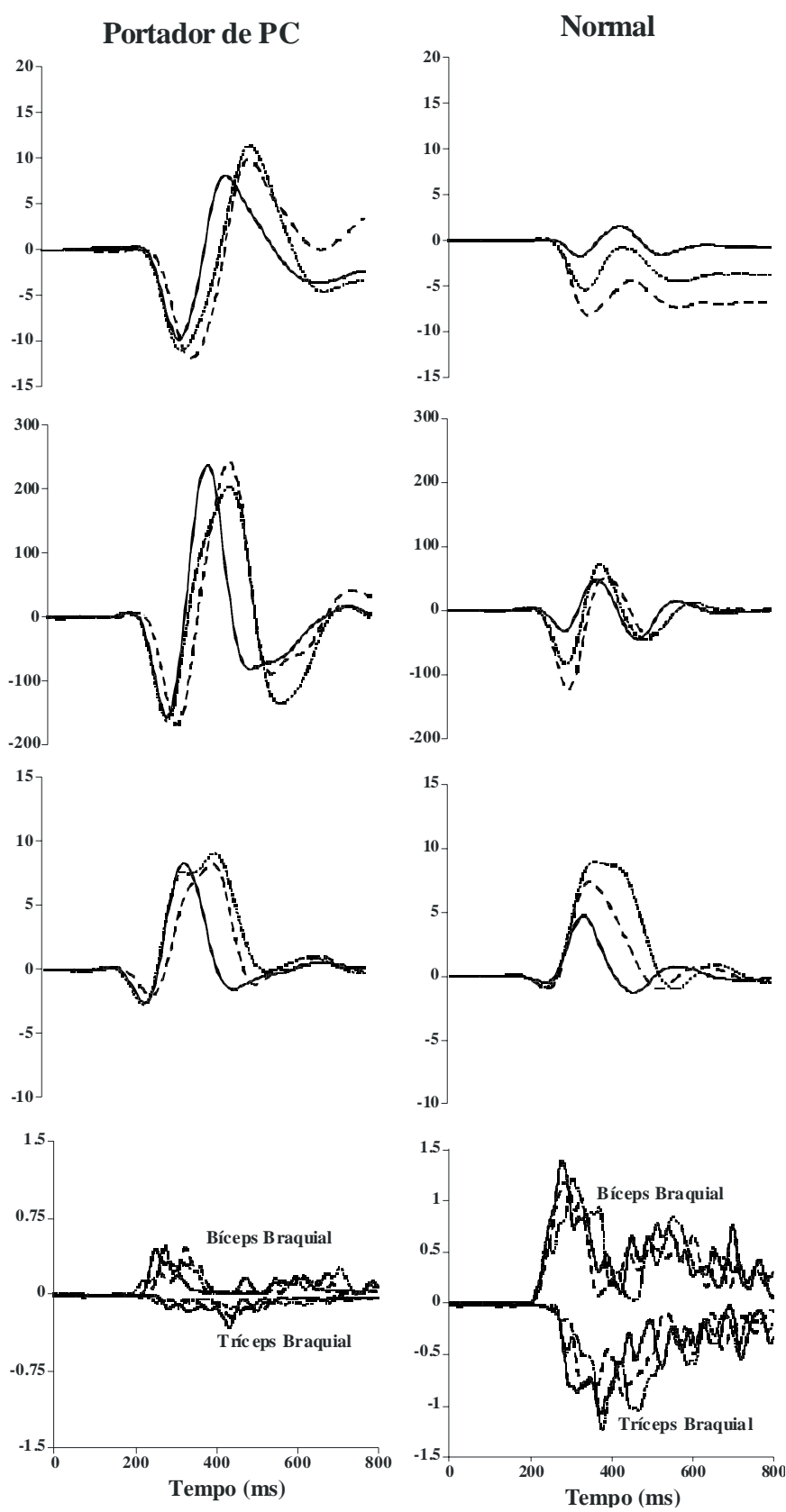

Figura 1. Deslocamento angular (em graus), velocidade angular (em graus por segundo) e torque muscular (em Newton·metros) do ombro (A) e do cotovelo (B), e atividade eletromiográfica (EMG) (em milivolts) dos músculos deltóide anterior e deltóide posterior (A), e bíceps braquial e tríceps braquial (B) na tarefa de flexão do ombro. Esses dados são referentes a uma repetição para cada distância angular (25, linha contínua; $50^{\circ}$, linha tracejada; e $75^{\circ}$, linha pontilhada), realizada por um portador de paralisia cerebral hemiplégica espástica (portador de PC) e um indivíduo sem comprometimento motor e/ou neurológico (Normal).

\section{Tarefa de flexão do cotovelo}

A Figura 2 ilustra os deslocamentos e as velocidades angulares, os torques musculares e os padrões de atividade EMG muscular referentes ao cotovelo (porção esquerda) e ao ombro (porção direita) durante a tarefa de flexão do cotovelo. Resultados qualitativos similares ao ombro na tarefa de flexão do ombro foram observados para o cotovelo na tarefa de flexão do cotovelo. Porém, nessa tarefa, um aspecto que pode ser observado nitidamente é que o portador de PC apresentou deslocamento angular do ombro maior que o 
(A) Cotovelo
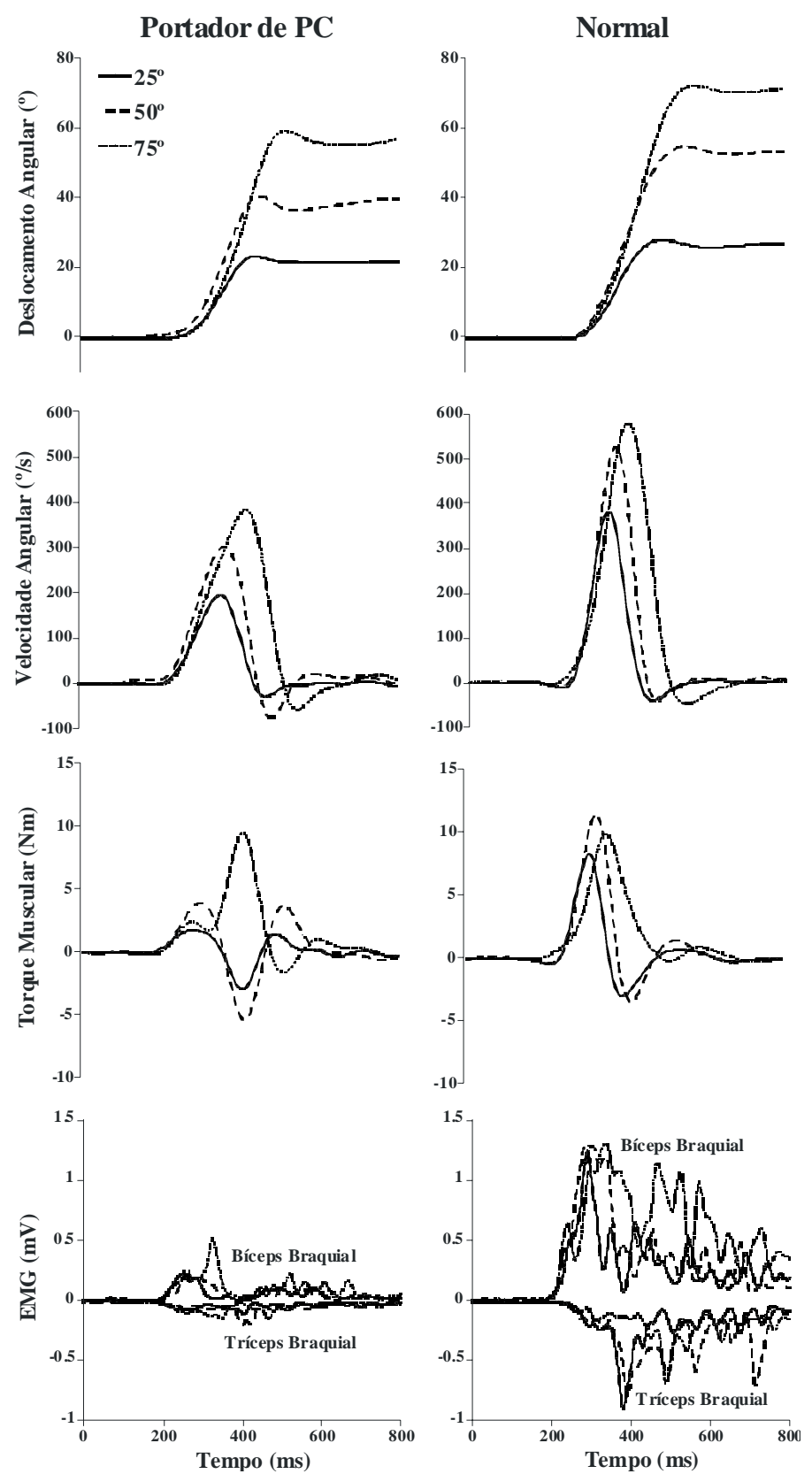

(B) Ombro
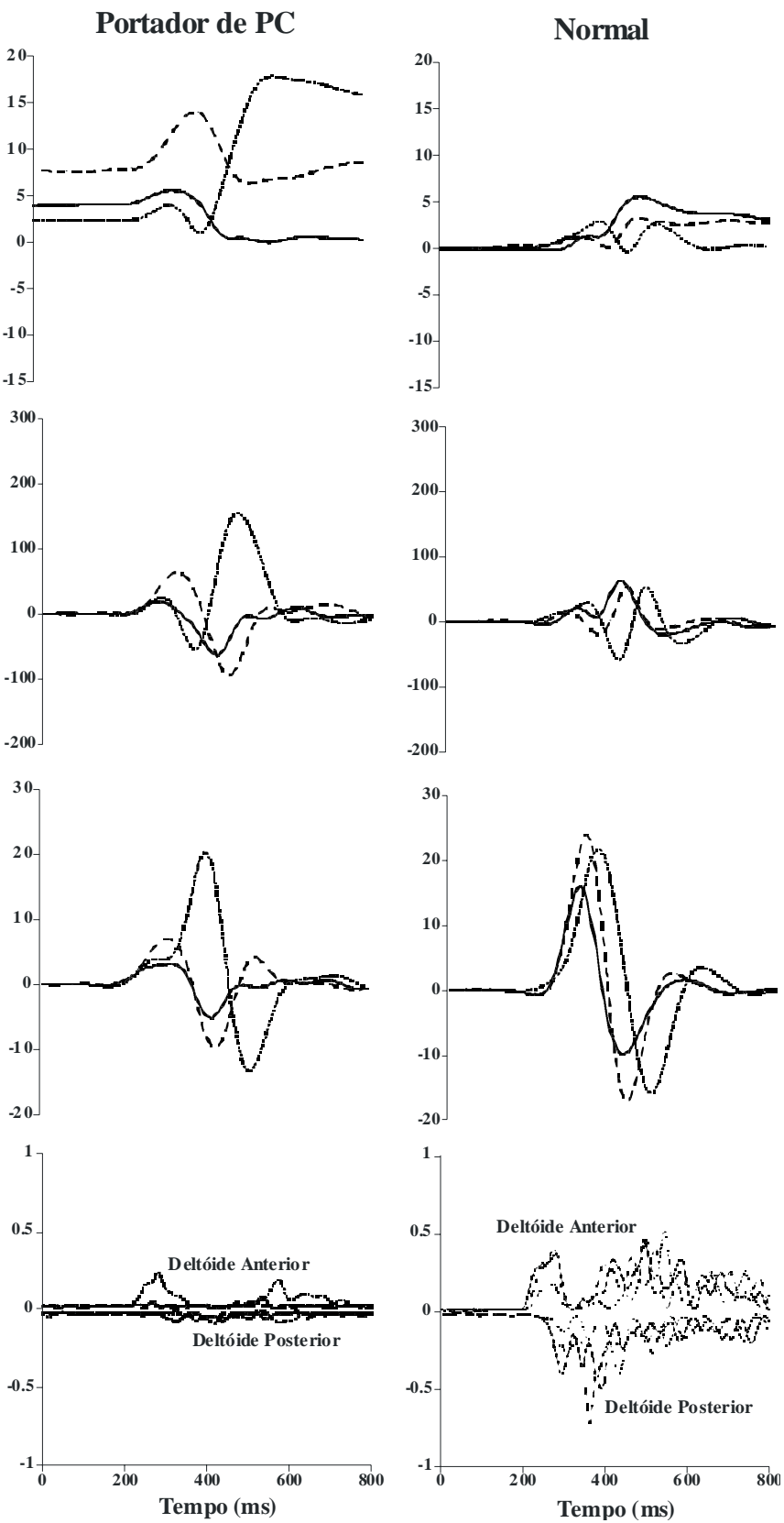

Figura 2. Deslocamento angular (em graus), velocidade angular (em graus por segundo) e torque muscular (em Newton·metros) do cotovelo (A) e do ombro (B), e atividade eletromiográfica (EMG) (em milivolts) dos músculos bíceps braquial e tríceps braquial (A), e deltóide anterior e deltóide posterior (B) na tarefa de flexão do cotovelo. Esses dados são referentes a uma repetição para cada distância angular $\left(25^{\circ}\right.$, linha contínua; $50^{\circ}$, linha tracejada; e $75^{\circ}$, linha pontilhada), realizada por um portador de paralisia cerebral hemiplégica espástica (portador de PC) e um indivíduo sem comprometimento motor e/ou neurológico (Normal).

indivíduo normal nas distâncias angulares de $50^{\circ}$ e $75^{\circ}$. O indivíduo normal, por sua vez, parece ter estendido o ombro no início do movimento (Figura 2, porção direita).

A Tabela 2 apresenta os valores médios ( \pm desvio padrão) das variáveis analisadas. A ADM do cotovelo e a velocidade máxima dessa articulação foram menores para os portadores de PC do que para os indivíduos normais e ambas aumentaram com o aumento da distância angular. Com relação à ADM do ombro, os portadores de PC apresentaram ADM dessa articulação maior que os indivíduos normais na distância 
angular de $75^{\circ}$. Ainda, os portadores de PC apresentaram impulso de aceleração do cotovelo menor que os indivíduos normais, e esse impulso aumentou com o aumento da distância angular. O impulso de desaceleração do cotovelo, por outro lado, não apresentou diferença entre as distâncias angulares para os dois grupos. Finalmente, apenas o impulso de aceleração do ombro que aumentou com o aumento da distância angular para os dois grupos, enquanto que nenhuma diferença foi encontrada para o impulso de desaceleração dessa articulação nessa tarefa (Tabela 2).

\section{DISCUSSÃO}

O objetivo geral deste estudo foi analisar os movimentos articulares do ombro e do cotovelo em portadores de PC (hemicorpo não plégico) e em indivíduos normais (hemicorpo direito) durante a execução das tarefas de flexão do ombro e do cotovelo. É importante salientar que foi solicitado a todos os participantes que movimentassem somente a articulação do ombro para tarefa de flexão de ombro e a do cotovelo para a tarefa de flexão do cotovelo.

Tabela 2. Média ( \pm desvio padrão) das variáveis amplitude de movimento do cotovelo e do ombro, velocidade máxima do cotovelo, impulsos de aceleração e de desaceleração do cotovelo e do ombro, intensidade de ativação e quantidade de atividade muscular do bíceps braquial e do deltóide anterior, e latência antagonista entre os músculos bíceps braquial e tríceps braquial, e deltóide anterior e deltóide posterior durante a tarefa de flexão de cotovelo dos portadores de PC e dos indivíduos normais nas três distâncias angulares $\left(25^{\circ}, 50^{\circ}\right.$ e $\left.75^{\circ}\right)$.

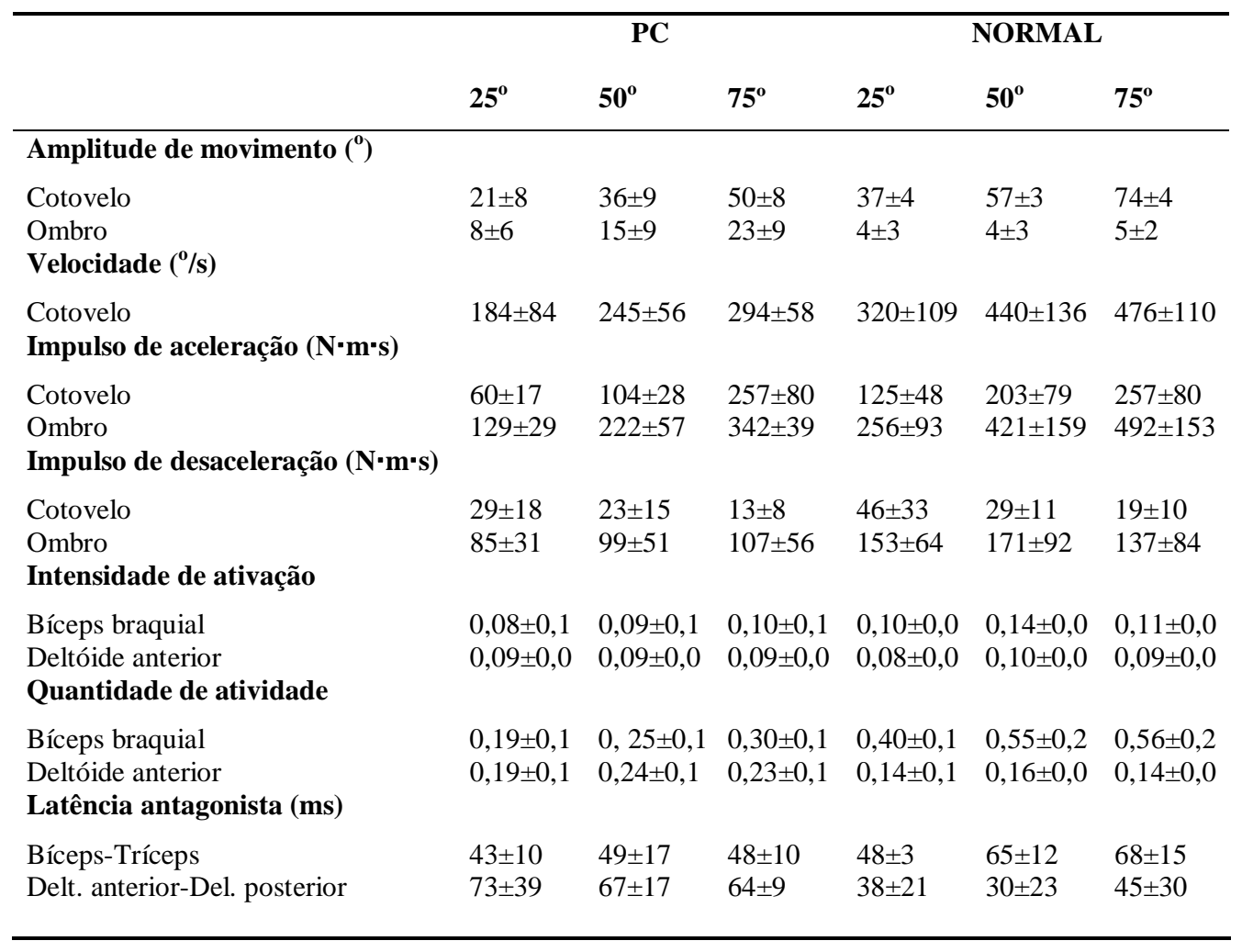

Em se tratando da atividade EMG muscular, $\mathrm{Q}_{30}$ do bíceps braquial e do deltóide anterior foi a mesma entre os grupos e as distâncias angulares. Entretanto, enquanto que os portadores de PC apresentaram $\mathrm{Q}_{\mathrm{ag}}$ do bíceps braquial menor que os indivíduos normais, nenhuma diferença foi encontrada para o músculo deltóide anterior. Por fim, os portadores de PC apresentaram $\mathrm{L}_{\text {ant }}$ entre os músculos bíceps braquial e tríceps braquial menor que os indivíduos normais, e apresentaram $\mathrm{L}_{\text {ant }}$ entre os músculos deltóide anterior e deltóide posterior maior que os indivíduos normais.
Os resultados encontrados neste estudo estão de acordo com o pressuposto teórico da hipótese da estratégia dual ${ }^{6}$ para a maioria das variáveis investigadas. Sendo assim, os participantes dos dois grupos recrutaram inicialmente o mesmo número de unidades motoras para realizar as duas tarefas motoras, e geraram uma quantidade de atividade dos músculos deltóide anterior e bíceps braquial maior para as distâncias angulares maiores para realizar as tarefas de flexão de ombro e de cotovelo, respectivamente, conforme estudo anterior ${ }^{13}$. Porém, enquanto que os portadores de PC modularam a 
quantidade de atividade muscular com o aumento da distância angular assim como os indivíduos normais, a quantidade média dessa atividade foi menor para realizar a tarefa de flexão do cotovelo. Ainda, a ADM das articulações do ombro (tarefa de flexão do ombro) e do cotovelo (tarefa de flexão do cotovelo) e a velocidade máxima foram maiores para as distâncias angulares maiores. Contudo, mais uma vez, os portadores de PC apresentaram valores menores para essas variáveis para realizar a tarefa de flexão do cotovelo.

Os resultados desse estudo não apontaram diferença no controle dos movimentos voluntários entre o hemicorpo não plégico dos portadores de PC hemiplégica espástica e os indivíduos normais para realizar a tarefa de flexão do ombro. Porém, o mesmo não é verdadeiro quando realizaram a tarefa de flexão do cotovelo. Para realizar essa tarefa, os portadores de PC utilizaram uma estratégia diferente de controle dos movimentos da dos indivíduos normais. O grande deslocamento na articulação do ombro durante a realização da tarefa de flexão de cotovelo pode indicar uma diminuição na capacidade de controlar movimentos mais distais, como sugerido por Gordon et al. ${ }^{1}$.

\section{CONCLUSÃO}

De acordo com os resultados deste estudo, pode-se concluir que o hemicorpo não plégico de portadores de PC hemiplégica espástica não deve ser considerado normal ${ }^{14}$ ou intacto $^{15}$, uma vez que os movimento nas partes mais distais foram controlados diferentemente das partes proximais, o que não ocorreu com indivíduos normais. Esse fato foi constatado no presente estudo e confirma estudos anteriores sobre o hemicorpo não plégico de portadores de PC hemiplégica espástica ${ }^{1,2}$. Finalmente, pode-se sugerir que ao propor qualquer tratamento ou intervenção para os portadores de PC, é importante considerar não apenas o hemicorpo plégico dessa população. Pelo contrário, os dois hemicorpos devem ser considerados, uma vez que esses indivíduos não apresentam as mesmas características em termos de controle motor como as verificadas em indivíduos normais.

Agradecimentos: À Coordenação de Aperfeiçoamento de Pessoal de Nível Superior (CAPES), pela bolsa de Mestrado, Programa de Demanda Social.

\section{REFERÊNCIAS BIOBLIOGRÁFICAS}

1. Gordon AM, Charles J, Duff SV. Fingertip forces during object manipulation in children with hemiplegic cerebral palsy. II: bilateral coordination. Dev Med Child Neurol 1999; 41(3): 17685.

2. Brown JV, Schumacher U, Rohlmann A, Ettlinger G, Schmidt C, Skreczek W. Aimed movements to visual target in hemiplegic and normal children: is the "good" hand of children with infantile hemiplegia also normal? Neuropsychologia 1989; 27: 283-302.

3. Latash ML. Control of human movement Champaing(IL): Human Kinetics; 1993.

4. Corcos DM, Gottlieb GL, Agarwal GC. Organizing principles for single-joint movements. II. A speed-sensitive strategy. J Neurophysiol 1989; 62(2): 358-68.

5. Gottlieb GL, Corcos DM, Agarwal GC. Organizing principles for single-joint movements. I. A speed-insensitive strategy. J Neurophysiol 1989; 62(2): 342-57.

6. Gottlieb GL, Corcos D, Agarwal GC. Strategies for the control of voluntary movements with one mechanical degree of freedom. Behav Brain Sci 1989; 12: 189-250.

7. Khan MA, Garry MI, Franks IM. The effect of target size and inertial load on the control of rapid aiming movements. A test of speed-sensitive and speed-insensitive strategies. Exp Brain Res 1999; 124(2): 151-8.

8. Almeida GL, Corcos DM, Latash ML. Practice and transfer effects during fast single-joint elbow movements in individuals with Down syndrome. Phys Ther 1994; 74(11): 1000-12.

9. Almeida GL, Campbell SK, Girolami GL, Penn RD, Corcos DM. Multidimensional assessment of motor function in a child with cerebral palsy following intrathecal administration of baclofen. Phys Ther 1997; 77(7): 751-64.

10. Barela AMF. Controle de movimentos voluntários em portadores de paralisia cerebral hemiplégica espástica [Dissertação de Mestrado]. Rio Claro(SP): Universidade Estadual Paulista; 2000.

11. Winter DA. Biomechanics and motor control of human movement. $2^{\text {nd }}$ ed. New York(NY): John Wiley \& Sons; 1990.

12. Hinkle DE, Wiersma W, Jurs SG. Applied statistics for the behavioral sciences. $3^{\text {rd }}$ ed. Boston(MA): Houghton Mifflin; 1994.

13. Almeida GL, Hong DA, Corcos D, Gottlieb GL. Organizing principles for voluntary movement: extending single-joint rules. J Neurophysiol 1995; 74(4): 1374-81.

14. Brown JK, van Rensburg F, Walsh G, Lakie M, Wright GW. A neurological study of hand function of hemiplegic children. Dev Med Child Neurol 1987; 29(3): 287-304.

15. Nezu A, Kimura S, Takeshita S, Tanaka M. Functional recovery in hemiplegic cerebral palsy: ipsilateral electromyographic responses to focal transcranial magnetic stimulation. Brain Dev 1999; 21(3): 162-5. 


\section{ERRATA/ERRATUM}

No volume 10.3, Julho-Setembro de 2006, na página 327, no item Análise Estatística, do artigo "Controle de movimentos voluntários no membro superior não plégico de portadores de paralisia cerebral hemiplégica espástica” onde se lê: Quando os fatores indicaram diferenças nas análises multivariadas, análises univariadas foram empregadas e indicaram diferença para distância angular e para interação entre grupo e distância angular, testes.. o correto é: "Quando os fatores indicaram diferenças nas análises multivariadas, análises univariadas foram empregadas e quando as análises univariadas indicaram diferença para distância angular e para interação entre grupo e distância angular, testes...” 
O arquivo disponível sofreu correções conforme ERRATA publicada no Volume 11 Número 1 da revista. 